\title{
THE DØ RUN II DETECTOR AND PHYSICS PROSPECTS
}

\author{
NEETI PARASHAR \\ 111 Dana Research Center, Northeastern University, 360 Huntington Avenue, \\ Boston, MA 02115, USA \\ E-mail: neeti@fnal.gov \\ FOR THE DØ COLLABORATION
}

\begin{abstract}
The D $\varnothing$ Detector at Fermilab is currently undergoing an extensive upgrade to participate in the Run II data taking which shall begin on March 1, 2001. The design of the detector meets the requirements of the high luminosity environment provided by the accelerator. This paper describes the upgraded detector subsystems and gives a brief outline of the physics prospects associated with the upgrade.
\end{abstract}

\section{Introduction}

The $\mathrm{D} \varnothing$ detector was designed to study collisions between protons and antiprotons in the Tevatron collider at Fermilab. D $\varnothing$ took its first data run in the period 1992-1996, called Run I. The successes of Run 1, including the discovery of the top quark, and the physics potential of high-luminosity running at the Tevatron have additionally motivated the present upgrade of the detector. We would now like to pursue a detailed top quark physics study, search for the Higgs boson and for supersymmetry, investigate b-physics, and look for physics beyond the Standard Model (SM).

In order to enhance the physics reach of these processes in Run II, the Tevatron also had to undergo two major upgrades. First, the Tevatron for Run II is designed to achieve a luminosity of $5 \times 10^{32} \mathrm{~cm}^{-2} \mathrm{~S}^{-1}$, which is a factor of 10 more than in Run I. The second upgrade involves a decrease in the bunch crossing time, which for Run I was $3.5 \mu s$, while Run II will begin with $396 \mathrm{~ns}$ and eventually reach $132 \mathrm{~ns}$ as the number of bunches is increased. There is also an increase in the center-of-mass (CM) energy from $1.8 \mathrm{TeV}$ to $2.0 \mathrm{TeV}$. To take full advantage of the new physics opportunities and to contend with the high radiation environment and shorter bunch crossing times, an extensive upgrade of the $\mathrm{D} \varnothing$ detector was undertaken, which is now in its final stages.

Figure 1 shows an elevation view of the upgraded detector. The upgrade consists of an addition of a solenoid, a tracker with silicon and fiber detectors, muon scintillation trigger counters, new forward muon system, preshower detectors, readout electronics and new trigger and data acquisition systems.

ws-p8-50x6-00: submitted to World Scientific on November 13, 2018 


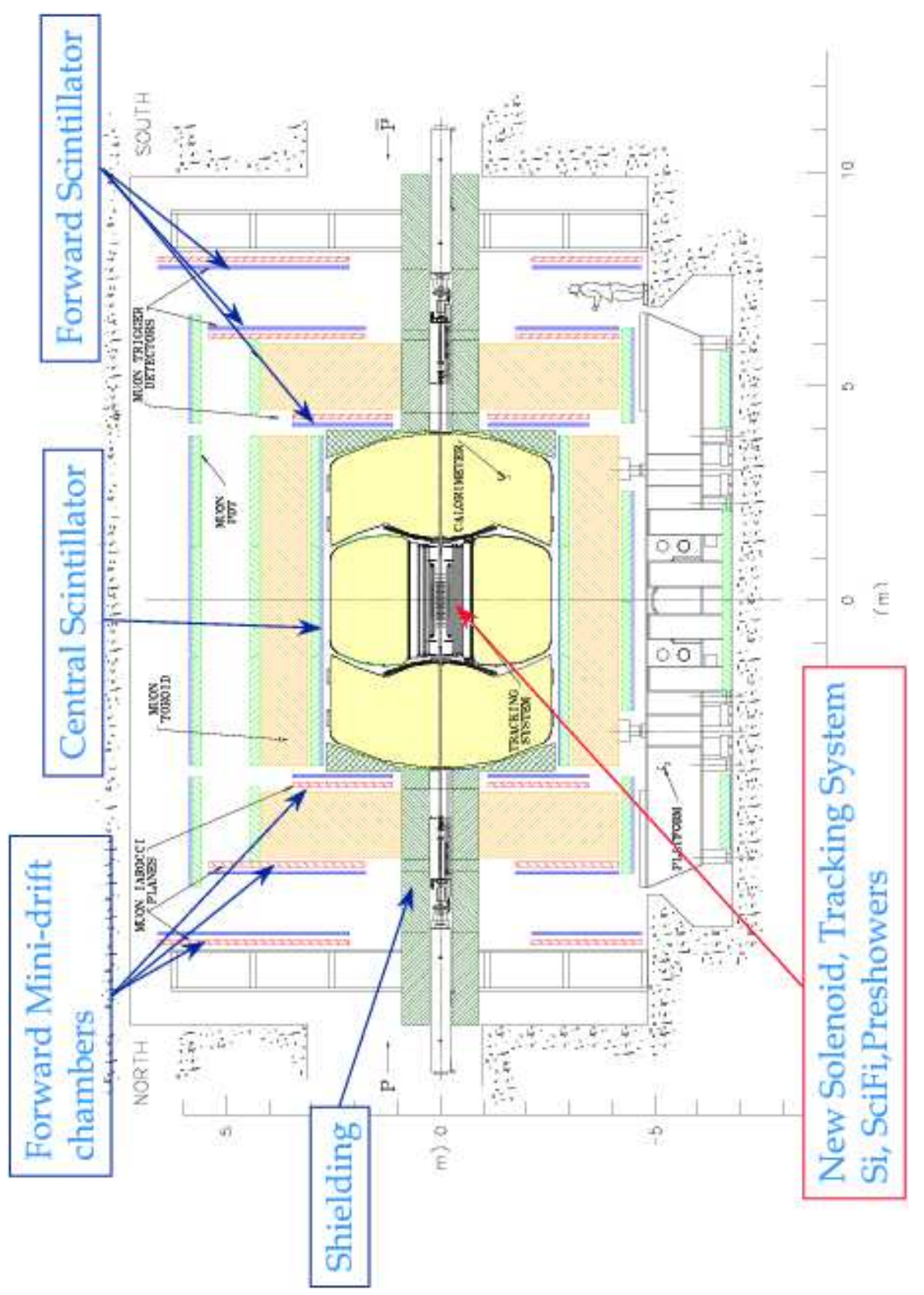

Figure 1. Elevation view of the upgraded D $\varnothing$ detector for Run II. 


\section{Tracking}

The upgraded tracking system (Figure 2) consists of an inner silicon vertex detector, surrounded by eight superlayers of scintillating fiber tracker. These detectors are located inside a 2 Tesla superconducting solenoid, which is surrounded by a scintillator based preshower detector. The upgraded tracking system has been designed to meet several goals: momentum measurement by the introduction of a solenoidal field; good electron identification and $e / \pi$ rejection; tracking over a large range in pseudo-rapidity $(\eta \approx \pm 3)$; secondary vertex measurement for identification of $b$-jets from Higgs and top decays and for $b$-physics; first level tracking trigger; fast detector response to enable operation with a bunch crossing time of $132 \mathrm{~ns}$; and radiation hardness.

\subsection{Silicon Microstrip Tracker (SMT)}

The silicon tracker is the first set of detectors encountered by particles emerging from the collision. The detector design consists of interspersed disks and barrels, based on single and double-sided silicon microstrip detectors, with a total of 793,000 channels and is radiation hard up to about $2 \mathrm{Mrad}$. The SVX IIe chip is used for readout 1 . The combination of small-angle and largeangle stereo provides good pattern recognition and allows good separation of primary vertices in multiple interaction events. The expected hit position resolution in $r \phi$ is $10 \mu m$.

A silicon track trigger preprocessor is being built which will allow the use of SMT information in the Level 2 trigger. This will add the capability for triggering on tracks displaced from the primary vertex, as well as sharpening the $p_{T}$ threshold of the Level 2 track trigger and of the electron and jet triggers at Level 3.

\subsection{Central Fiber Tracker (CFT)}

The detector just outside the SMT is the 8-layered CFT, which is based on scintillating fiber ribbon doublets with visible light photon counter (VLPC) readout 1 . Each layer contains 2 fiber doublets in a $z u$ or $z v$ configuration ( $z=$ axial fibers and $u, v= \pm 3^{\circ}$ stereo fibers). Each doublet consists of two layers of $830 \mu \mathrm{m}$ diameter fibers with $870 \mu \mathrm{m}$ spacing, offset by half the fiber spacing. This configuration provides very good efficiency and pattern recognition and results in a position resolution of $\approx 100 \mu \mathrm{m}$ in $r \phi$. There are a total of 77,000 channels.

The CFT serves two main functions. First, with the SMT it enables track reconstruction and momentum measurement for $\eta< \pm 1.7$, second it provides

ws-p8-50x6-00: submitted to World Scientific on November 13, 2018 


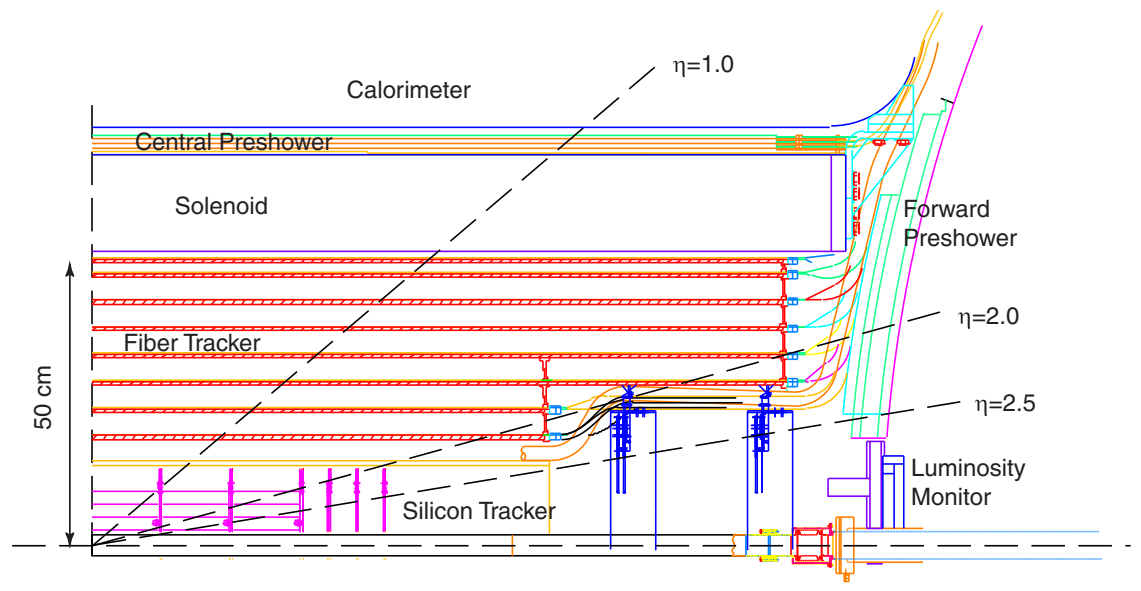

Figure 2. $r-z$ view of the $\mathrm{D} \varnothing$ tracking system.

fast Level 1 triggering on charged track momentum.

The fibers are up to $2.5 \mathrm{~m}$ long and the light is piped out by clear fibers of length 7-11 $\mathrm{m}$ to the VLPCs situated in cryostats outside the tracking volume, which are maintained at $9^{\circ} \mathrm{K}$. The VLPCs are solid state devices with a pixel size of $1 \mathrm{~mm}$, matched to the fiber diameter. The fast risetime, high gain and excellent quantum efficiency of these devices make them ideally suited to this application.

\subsection{Superconducting Solenoid}

The momenta of the charged particles will be determined from their curvature in the $2 \mathrm{~T}$ magnetic field provided by a $2.8 \mathrm{~m}$ long, $1.42 \mathrm{~m}$ in diameter and 1.1 radiation length thick solenoid magnet. From the value of $\sin \theta \times \int B_{z} d l$ and the space point precision provided by the silicon and fiber tracking system (located inside the solenoid) a momentum resolution of $\delta p_{T} / p_{T}=0.002 p_{T}$, $\left(p_{T}\right.$ in $\left.\mathrm{GeV}\right)$ is expected.

\subsection{Preshower detectors}

The central and forward preshower detectors (CPS and FPS) are based on a similar technology, using triangular scintillator strips (axial and $20^{\circ}$ stereo) with wavelength shifter readout. These detectors provide fast energy and 
position measurements for the electron trigger and facilitate offline electromagnetic identification.

\section{Muon Detectors}

The higher event rates in Run II have led us to add new muon trigger detectors covering full pseudorapidity range and the harsh radiation environment has prompted us to replace the forward proportional drift tubes(PDTs) with minidrift tubes (MDTs). In the central region, the Run I PDTs are used and the front-end electronics have been replaced to ensure dead-timeless operation.

The scintillation counters provide the time information and match the muon tracks in the fiber tracker, and consists of three layers to reduce hit combinatorics. The drift tubes provide enhanced muon momentum resolution and pattern recognition. The design of the muon system reduces backgrounds and trigger rates with additional shielding and we have the ability to trigger on inclusive single muons with $p_{T}>7 \mathrm{GeV}$ and dimuons with $p_{T}>2 \mathrm{GeV}$.

\section{Trigger and Data Acquisition}

The DØ trigger and DAQ systems have been completely restructured to handle the shorter bunch spacing and new detector subsystems in Run II. The Level 1 and 2 triggers utilize information from the calorimeter, preshower detectors, central fiber tracker, and muon detectors. The Level 1 trigger reduces the event rate from $7.5 \mathrm{MHz}$ to $10 \mathrm{KHz}$ and has a latency of $4 \mu \mathrm{sec}$. The trigger information is refined at Level 2 using calorimeter clustering and detailed matching of objects from different subdetectors. The Level 2 trigger has an output rate of $1 \mathrm{KHz}$ and a latency of $100 \mu \mathrm{sec}$. Level 3, consisting of an array of PC processors, partially reconstructs event data within 50 msec to reduce the rate to $50 \mathrm{~Hz}$. Events are then written to tape.

\section{Run II Physics Prospects - Some Highlights}

We have seen that the design of the Run II DØ detector makes important additions to the $\mathrm{D} \varnothing$ physics capabilities, namely: energy/momentum matching for electron identification, improved muon momentum resolution, charged sign and momentum determination, calorimeter calibration and displaced vertex identification (b-tags).

One of the crucial physics goals of Run II is to search for the Higgs boson. In Run II the reach for Higgs at the Tevatron suggests a possibility of discovering light Higgs, given sufficient integrated luminosity from both experiments

ws-p8-50x6-00: submitted to World Scientific on November 13, 2018 


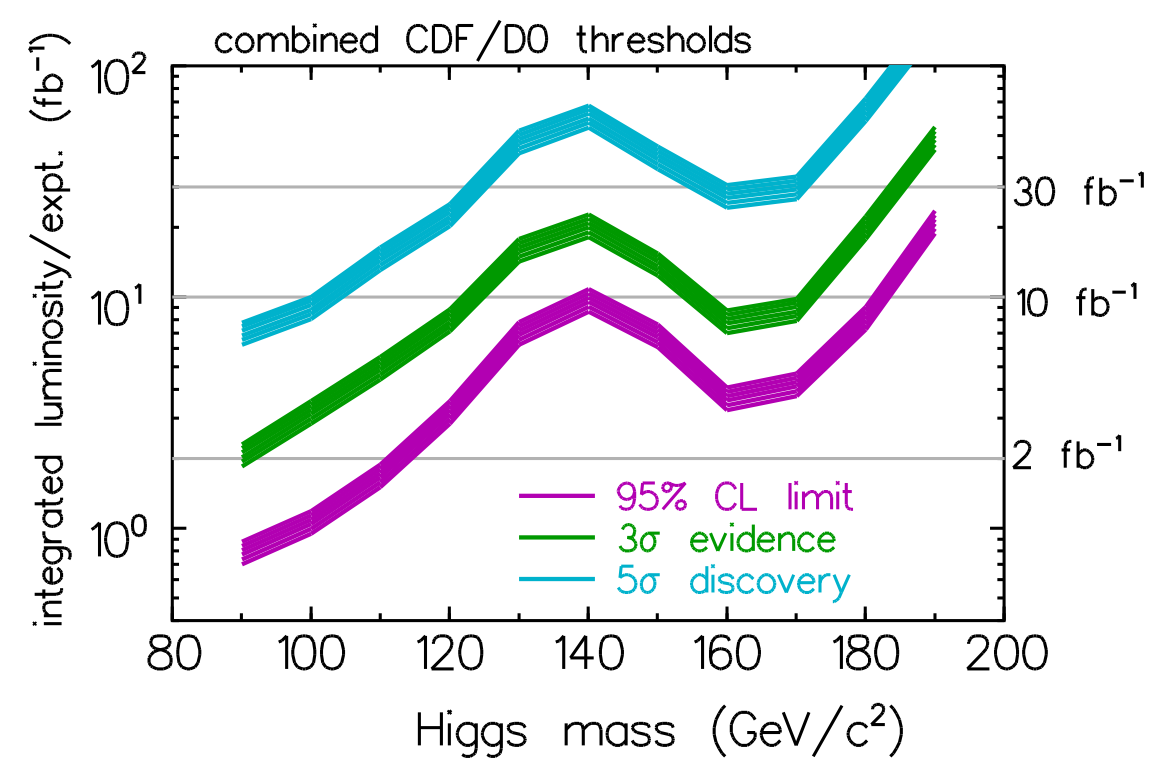

Figure 3. Integrated luminosity required as a function of Higgs mass for a 95\% C.L. exclusion, $3 \sigma$ evidence and $5 \sigma$ discovery.

(DØ and Collider Detector at Fermilab, CDF) at the collider 3 . The dominant decays can be classified into $M_{\text {Higgs }}<135 \mathrm{GeV}$ and $M_{\text {Higgs }}>135 \mathrm{GeV}$. The light mass range is of great interest, since minimal supersymmetric versions of SM always predict a light Higgs boson lying in this region. If there is no SM Higgs, $95 \%$ confidence level exclusion limits can be set up to $\approx 120 \mathrm{GeV}$ mass with $2 \mathrm{fb}^{-1}$. However if there is a Higgs, discovery at the $5 \sigma$ level can be obtained with $20 \mathrm{fb}^{-1}$ up to similar mass. This sensitivity is shown in Figure 3 .

A detailed study of the top quark is another important physics interest. Since the CM energy is expected to increase from $1.8 \mathrm{TeV}$ to $\approx 2.0 \mathrm{TeV}$, the $t \bar{t}$ production cross section will increase by about $38 \%$ (the production is dominated by $q \bar{q} \rightarrow t \bar{t}$ ). For single top production the increase will be about $22 \%$ for the $s$-channel $(q \bar{q} \rightarrow t \bar{b})$, and about $44 \%$ for the $W$-gluon fusion process $(q g \rightarrow q t \bar{b})$. The expected uncertainty in the top quark mass measurement is $\delta m_{t} \simeq 2 \mathrm{GeV}$, and we should also be able to observe single top production for the first time.

SM has been very successful so far and in the electroweak sector, the goal

ws-p8-50x6-00: submitted to World Scientific on November 13, 2018 
is to make more precise tests of the SM and search for deviations that may signal the presence of new physics. A prime measurement is of the $\mathrm{W}$ mass, which in Bun II would be done with a precision of $30 \mathrm{MeV}$ from both $\mathrm{D} \varnothing$ and CDF $\mathrm{E}$. Within SM, the mass of the top quark and the $\mathrm{W}$ boson set constraints on the mass of the Higgs boson.

In physics beyond the SM, there is potential for observing supersymmetry. For example, the supersymmetric gaugino pair production in the trilepton decay modes $p \bar{p} \rightarrow \tilde{\chi}_{1}^{ \pm} \tilde{\chi}_{1}^{\mp}, \tilde{\chi}_{1}^{ \pm} \tilde{\chi}_{2}^{0} \rightarrow 3 \ell+X$, can be discovered with a mass reach of $220 \mathrm{GeV}$. Supersymmetric squark/gluino production in the jets + $E_{T}$ channel is expected to probe masses up to about $400 \mathrm{GeV}$ for $2 \mathrm{fb}^{-1}$. Other interesting searches include a wide range of topics, namely, leptoquarks, new gauge bosons $\left(W^{\prime}, Z^{\prime}\right)$, topcolor, compositeness, technicolor and extra dimensions. In Run II there is an excellent chance to discover new physics or exclude significant regions of parameter space.

A wide range of b-physics and QCD physics will also be pursued, but their details are beyond the scope of this article. Details of the D $\varnothing$ detector and its upgrade can be found elsewhere $\mathrm{E}$.

\section{Acknowledgments}

I would like to thank the Lake Louise Winter Institute for arranging a stimulating set of talks and for their warm hospitality. I would also like to thank Darien Wood for careful reading of this paper.

\section{References}

1. T. Zimmerman et al, IEEE Trans. Nucl. Sci. 42, 803 (1995).

2. M.D. Petroff and M.G. Stapelbroek, IEEE Trans. Nucl. Sci. 36, 158 (1989);M.D. Petroff and M. Atac, IEEE Trans. Nucl. Sci. 36, 163 (1989).

3. M. Carena et al, Report of the Tevatron Higgs Working Group, hep$\mathrm{ph} / 0010338$.

4. R. Brock et al, Report of the Working Group on Precision Measurements, hep-ex/0011009.

5. Report on "The D $\varnothing$ upgrade: The Detector and its Physics, Fermilab Pub-96/357-E; J. Ellison, "The D $\varnothing$ Detector Upgrade and Physics Program, hep-ex/0101048. 